\title{
Altered distribution of the EphA4 kinase in hippocampal brain tissue of patients with Alzheimer's disease correlates with pathology
}

\author{
Andrea FN Rosenberger ${ }^{1,2^{*}}$, Annemieke JM Rozemuller ${ }^{2}$, Wiesje M van der Flier ${ }^{1}$, Philip Scheltens ${ }^{1}$,
} Saskia M van der Vies ${ }^{2}$ and Jeroen JM Hoozemans ${ }^{2}$

\begin{abstract}
Synaptic dysfunction occurs early in the progression of Alzheimer's disease (AD) and correlates with memory decline. There is emerging evidence that deregulation of Erythropoietin-producing hepatocellular (Eph) receptor tyrosine kinases (RTK) signaling contributes to the aberrant synaptic functions associated with neurodegeneration. The Eph receptor A4 is highly expressed in human adult hippocampal brain tissue and was previously linked to cognitive impairment in a transgenic mouse model for AD. Whether EphA4 levels are altered in AD brain remains elusive. Therefore we investigated the protein levels and localization of EphA4 in human hippocampus derived from AD $(n=29)$ as well as non-demented control cases $(n=19)$.

The total EphA4 protein levels were not changed in AD patients compared to control cases. However, immunohistochemical localization of EphA4 revealed an altered distribution in AD compared to control hippocampus. EphA4 immunoreactivity was observed in plaque-like structures in AD cases. Double-labelling with phosphorylated tau and amyloid beta indicates that EphA4 co-localizes with neuritic plaques in AD. This altered distribution pattern was observed at early stages (Braak stage II) and correlates with the hallmarks of AD pathology suggesting a reduced availability of EphA4 that is likely to contribute to synaptic dysfunction that occurs early in AD.
\end{abstract}

Keywords: Alzheimer's disease, EphA4 kinase, Synapse, Immunohistochemistry

\section{Introduction}

Alzheimer's disease $(\mathrm{AD})$ is the most common neurodegenerative disorder and has an increasing effect on our ageing population. Pathological hallmarks of $\mathrm{AD}$ are extracellular amyloid beta $(A \beta)$ deposits and intracellular accumulation of hyper-phosphorylated tau protein leading to the formation of neurofibrillary tangles (NFTs) [1]. In addition, progressive synaptic dysfunction is thought to occur in early stages of the disease and has been found to correlate closely with cognitive deficits observed in patients with AD [2-4]. There is emerging evidence that the erythropoietin-producing hepatocellular (Eph) receptors and their ligands, the so-called

\footnotetext{
* Correspondence: a.rosenberger@vumc.nl

'Alzheimer center \& Department of Neurology, Neuroscience Campus Amsterdam, VU University Medical Center, De Boelelaan 1118, $1081 \mathrm{HZ}$ Amsterdam, the Netherlands

${ }^{2}$ Department of Pathology, Neuroscience Campus Amsterdam, VU University Medical Center, De Boelelaan 1117, 1081 HV Amsterdam, the Netherlands
}

ephrins, are involved in aberrant synaptic functions associated with cognitive impairment in AD [5]. Eph/ ephrin signaling is required for a wide range of biological processes both during embryogenesis and adult life and involves the Eph receptors which form the largest of the 20 subfamilies of human receptor kinases. Eph/ephrin signaling plays a role not only during synapse formation and maturation and synaptic plasticity [6-8] in the brain but also in directing cell positioning and migration, axon guidance $[9,10]$, control of tissue morphogenesis, patterning, tumour invasion and metastasis, immune function [11,12], haematopoiesis and blood clotting [13] and tissue repair and maintenance.

Eph receptors and their ligands are exclusively membranebound and hence cell-cell contact is required for activation of the kinase through oligomerisation and transphosphorylation [14]. EphA4 is the Eph receptor family member that is most highly expressed in the adult hippocampus where it plays a role in adult synaptic plasticity and 
learning [15,16]. The EphA4 kinase is pre- and postsynaptically expressed on dendritic spines of pyramidal neurons and axon terminals [17]. Emerging evidence supports a critical role for EphA4/ephrin A3 signaling in the regulation of spine morphology in the hippocampus. Activation of EphA4 upon binding to its glia-derived ligand ephrin A3 was found to induce spine retraction and to trigger the reduction of dendritic spines and synaptic proteins, whereas inhibiting those interactions led to distorted spine shape and organization in the murine hippocampus. These findings suggest an essential role for EphA4 in the elimination of excitatory synapses [18-20].

Two major forms of $A \beta$ coexist in the brain: a shorter form with 40 amino acid residues and a longer form with 42 amino acids. The longer form is extremely toxic and can self-aggregate to form oligomers (amyloid beta oligomers, A $\beta O s$ ). Increased levels of EphA4 in cultured neurons and synaptoneurosomes was reported to be crucially involved in synaptic damage induced by A $\beta O s$ [21]. Interestingly, reduced expression of the EphA4 receptor has been linked to cognitive impairment in a transgenic mouse model for AD overexpressing the human amyloid beta precursor protein (APP) [22]. Loss of synapses is an early event in AD pathogenesis. It has therefore been suggested that changes in hippocampal EphA4 signaling might precede the onset of memory decline in AD. Whether EphA4 levels and activation are altered in human AD brain is not known.

In the present study we are the first group to report the involvement of EphA4 in AD pathology. We have investigated EphA4 expression levels and localization in human brain tissue of patients with $\mathrm{AD}$ and nondemented controls. An association of EphA4 with the hallmarks of AD was investigated using sequential single stainings and double-labelling with phosphorylated tau and amyloid beta.

\section{Material and methods Case selection}

Human brain tissue was obtained from the Netherlands Brain Bank (NBB, Amsterdam, The Netherlands). Prior to death, all donors gave written informed consent according to the Declaration of Helsinki for the use of their brain tissue and medical records for research purposes. This work was approved by the ethics committee of the NBB. Dementia status at death was determined on the basis of clinical information available during the last year of life. Neuropathological diagnosis was performed using histochemical analysis of formalin-fixed, paraffin-embedded tissue from different parts of the brain, including the frontal cortex (F2), temporal pole cortex, parietal cortex (superior and inferior lobule), occipital pole cortex and the hippocampus (essentially CA1 and entorhinal area of the parahippocampal gyrus) with routine stainings (hematoxilin and eosin, periodic acid Schiff-Luxol fast blue). Hippocampus and cortical areas were also stained with methenamine silver [23] and using the Bodian staining. Immunohistochemistry was performed using antibodies raised against hyperphosphorylated tau (AT8) and Abeta. Staging of AD pathology was evaluated according to modified assessment of Braak and Alafuzoff [24]. Cases with and without clinical neurological disease diagnosis were processed identically. Patients with co-morbidities like Parkinson's disease or Lewy-body disease were excluded from the study. Age, sex, clinical diagnosis and Braak score for neurofibrillary tangles (NFTs) of all cases used in study are listed in Table 1. Post mortem delay of all cases was between $2 \frac{1}{2}$ and 9 hours. In total, 29 patients with AD and 19 controls were included (see Table 1), with an average age at death of 84 years (range 57 to 100 years old); of these, 22 (45.8\%) were men.

\section{Western Blotting}

Frozen hippocampal tissue slides of patients in all Braak stages $(\mathrm{I}-\mathrm{VI}, \mathrm{n}=29)$ were cut and lysed with M-PER ( $\mathrm{pH}$ 7.6, Thermo Scientific) containing protease and phosphatase inhibitors (Roche). After incubation for $30 \mathrm{~min}$ on ice and subsequent centrifugation $(2 \times$ $\left.10 \mathrm{~min}, 4^{\circ} \mathrm{C}, 15682 \times \mathrm{g}\right)$, protein concentrations of the supernatants were determined with the standard Bradford Lowry Assay (Bio-Rad Protein Assay).

Protein lysates were re-suspended in sample buffer (Thermo Scientific) and heated for 5 minutes at $95^{\circ} \mathrm{C}$. Proteins were resolved by SDS-PAGE using $8-16 \%$ gradient polyacrylamide gels (mini-PROTEAN ${ }^{\circledR} \mathrm{TGX}^{\mathrm{\tau}}$, Bio-Rad) in running buffer (25 mM Tris, $192 \mathrm{mM}$ glycine, $0.1 \%$ SDS, pH 8.3, Bio-Rad) and electrophoretically transferred onto a nitrocellulose membrane $(0.2 \mu \mathrm{m}$; Whatman, Protran $^{\mathrm{Tm}}$ ) in transfer buffer (25 mM Tris, $192 \mathrm{mM}$ glycine, 20\% methanol, Bio-Rad). Membranes were blocked for 1 hour in Tris-buffered saline (50 mM Tris $\mathrm{pH} 7.5$, $0.15 \mathrm{M} \mathrm{NaCl}, 0.1 \%$ Tween-20) containing 5\% BSA (Roche Diagnostics) and incubated over night with primary anti-EphA4 antibody (1:500). A mouse monoclonal antibody directed at the extracellular (c-terminal) domain of the Ephrin-A4 receptor (amino acids 379-472; BD Transduction Laboratories) and a rabbit polyclonal EphA4 antibody directed at the intracellular domain (amino acids 890-904; Abcam) were utilised. Incubation with a secondary antibody linked to horseradish peroxidase ([HRP]-anti-rabbit IgG or HRP-anti-mouse IgG, 1:1000, DAKO) for 1 hour followed. Immunoreactive bands were detected with an enhanced chemiluminescence reagent (ECL Plus, GE Healthcare). Actin (mouse anti-actin AC-40, Sigma) was used as a loading control. The intensity of the resulting protein bands was quantified using MacBiophotonics ImageJ (version $1.47 \mathrm{k}$ ). 
Table 1 Cases used in this study

\begin{tabular}{|c|c|c|c|c|c|c|}
\hline Case no. & $\begin{array}{l}\text { Pathological } \\
\text { diagnosis }\end{array}$ & $\begin{array}{l}\text { Braak } \\
\text { stage }\end{array}$ & $\begin{array}{l}\text { Braak } \\
\text { (amyloid) }\end{array}$ & Sex & Age & PMD \\
\hline \multicolumn{7}{|c|}{ Immunohistochemistry } \\
\hline 1 & CTRL & 1 & $C$ & $M$ & 77 & $07: 30$ \\
\hline 2 & CTRL & 1 & B & $\mathrm{F}$ & 77 & $02: 55$ \\
\hline 3 & CTRL & 1 & B & $\mathrm{F}$ & 83 & 04:05 \\
\hline 4 & CTRL & 1 & B & $\mathrm{F}$ & 85 & 07:05 \\
\hline 5 & CTRL & 1 & B & M & 80 & $03: 18$ \\
\hline 6 & CTRL & 1 & 0 & $\mathrm{~F}$ & 81 & $04: 25$ \\
\hline 7 & CTRL & 2 & 0 & $\mathrm{~F}$ & 87 & 05:05 \\
\hline 8 & CTRL & 2 & 0 & $\mathrm{~F}$ & 76 & $05: 45$ \\
\hline 9 & CTRL & 2 & 0 & $\mathrm{~F}$ & 93 & $05: 50$ \\
\hline 10 & CTRL & 2 & B & $\mathrm{F}$ & 89 & $06: 25$ \\
\hline 11 & CTRL & 2 & B & $M$ & 79 & 09:00 \\
\hline 12 & CTRL & 2 & 0 & $M$ & 81 & 07:55 \\
\hline 13 & CTRL & 3 & C & $\mathrm{F}$ & 89 & $15: 40$ \\
\hline 14 & $A D$ & 3 & B & $M$ & 84 & $05: 20$ \\
\hline 15 & $A D$ & 3 & A & $\mathrm{F}$ & 98 & 06:05 \\
\hline 16 & $A D$ & 3 & B & $\mathrm{F}$ & 96 & 04:00 \\
\hline 17 & $A D$ & 3 & $C$ & $M$ & 78 & $05: 10$ \\
\hline 18 & $A D$ & 3 & $C$ & $\mathrm{~F}$ & 82 & 06:05 \\
\hline 19 & $A D$ & 4 & $C$ & $\mathrm{~F}$ & 84 & $04: 50$ \\
\hline 20 & $A D$ & 4 & $C$ & $M$ & 88 & 05:00 \\
\hline 21 & $A D$ & 4 & $C$ & $\mathrm{~F}$ & 87 & $06: 40$ \\
\hline 22 & $A D$ & 4 & $C$ & $\mathrm{~F}$ & 100 & $05: 15$ \\
\hline 23 & $A D$ & 4 & C & $M$ & 77 & 05:00 \\
\hline 24 & $A D$ & 5 & $C$ & $\mathrm{~F}$ & 84 & $04: 30$ \\
\hline 25 & $A D$ & 5 & $C$ & $\mathrm{~F}$ & 77 & $03: 45$ \\
\hline 26 & $A D$ & 5 & $C$ & $\mathrm{~F}$ & 78 & $08: 25$ \\
\hline 27 & $A D$ & 5 & $C$ & $M$ & 77 & 05:39 \\
\hline 28 & $A D$ & 5 & $C$ & $M$ & 86 & $06: 15$ \\
\hline 29 & $A D$ & 5 & B & $M$ & 69 & $07: 10$ \\
\hline 30 & $A D$ & 6 & $C$ & $M$ & 62 & $06: 45$ \\
\hline 31 & $A D$ & 6 & $C$ & $\mathrm{~F}$ & 66 & $05: 20$ \\
\hline 32 & $A D$ & 6 & $C$ & $\mathrm{~F}$ & 62 & - \\
\hline 33 & $A D$ & 6 & $C$ & $M$ & 57 & $07: 40$ \\
\hline 34 & $A D$ & 6 & $C$ & $\mathrm{~F}$ & 84 & $04: 50$ \\
\hline 35 & $A D$ & 6 & $C$ & $M$ & 65 & 06:00 \\
\hline \multicolumn{7}{|c|}{ Double stainings } \\
\hline 36 & $A D$ & 4 & C & $\mathrm{F}$ & 93 & $02: 30$ \\
\hline 37 & $A D$ & 5 & C & $M$ & 93 & $04: 30$ \\
\hline 38 & $A D$ & 5 & C & $\mathrm{F}$ & 81 & - \\
\hline 39 & $A D$ & 6 & C & $M$ & 74 & $05: 35$ \\
\hline 40 & CTRL & 1 & 0 & M & 96 & $06: 30$ \\
\hline 41 & CTRL & 1 & $A$ & $\mathrm{~F}$ & 94 & 04:05 \\
\hline
\end{tabular}

Table 1 Cases used in this study (Continued)

\begin{tabular}{lllllll}
\hline 42 & CTRL & 2 & A & M & 88 & $04: 43$ \\
43 & CTRL & 1 & B & F & 77 & $02: 55$ \\
Additional frozen tissue lysates & & & & \\
44 & AD & 4 & $C$ & $M$ & 84 & $03: 40$ \\
45 & AD & 4 & $C$ & $M$ & 81 & $04: 50$ \\
46 & AD & 6 & $C$ & $M$ & 57 & $03: 50$ \\
47 & CTRL & 1 & 0 & $M$ & 58 & $05: 15$ \\
48 & CTRL & 1 & B & F & 83 & $05: 30$ \\
\hline CTRL, control case; AD, Alzheimer's disease case; PMD, post mortem delay; F, \\
female; M, male.
\end{tabular}

Data is expressed as relative signal intensities (EphA4/ Actin) of the individual samples (Figure 1). Statistical analyses were performed by t-test analysis for independent samples using GraphPad Prism version 6.0 (San Diego, CA). Furthermore, whole protein extracts were analysed by Immuno-blot analysis with SDS-PAGE using precast stainfree gradient gels (Bio-Rad; Additional file 1: Figure S6). After UV activation of the gel, proteins were visualised and relative protein amounts were determined and used for normalization. Recombinant proteins EphA1 (75 kDa, aa 569-976; Pro Quinase), EphA4 (72 kDa, aa 586-986; Carna Biosciences) and EphB2 (73 kDa, aa 581987; Carna Biosciences) were used to proof specificity of the rabbit polyclonal EphA4 antibody. The EphA4 mouse antibody binds extracellularly (aa 379-472) which is outside of the binding domain for recombinant EphA4, therefore only the polyclonal rabbit antibody is shown (Additional file 2: Figure S8).

\section{Immunohistochemistry}

For single immunohistochemical analysis, formalin fixed (4\%, 24 hours) paraffin embedded $5 \mu \mathrm{m}$ thick sequential sections were mounted on coated glass slides (Menzel Gläser Superfrost PLUS, Thermo Scientific, Braunschweig Germany), dried over night at $37^{\circ} \mathrm{C}$ and deparaffinised. In order to block the endogenous peroxidase, the sections were incubated in $0.3 \%(\mathrm{v} / \mathrm{v}) \mathrm{H}_{2} \mathrm{O}_{2}$ in methanol (100\%) for $30 \mathrm{~min}$ at RT. Sections were boiled in $10 \mathrm{mM}$ sodium citrate buffer $(\mathrm{pH}$ 6.0) for 10 minutes for antigen retrieval. They were incubated over night with the primary antibody at room temperature. Mouse monoclonal anti-EphA4 [25-27] (1:200, BD Transduction Laboratories $\left.{ }^{\mathrm{rm}}\right)$; mouse monoclonal anti-phospho-tau [28] (AT8 1:200, Pierce Biotechnology, Rockford IL, USA); mouse monoclonal anti-A $\beta$ (IC16 1:500, Prof. C. Korth, Heinrich Heine University Düsseldorf, Germany) and mouse monoclonal anti-A $\beta$ 1-17 (VU17 [29] 1:1000, Dr. R. Veerhuis, VU University Medical Center, Amsterdam, NL) were used. As a second step, envision mouse/rabbit HRP (DAKO) was used. Sections were stained using the EnVision method (incubation with Dako REAL ${ }^{\mathrm{Tm}}$ EnVison $^{\mathrm{mm}}$ 


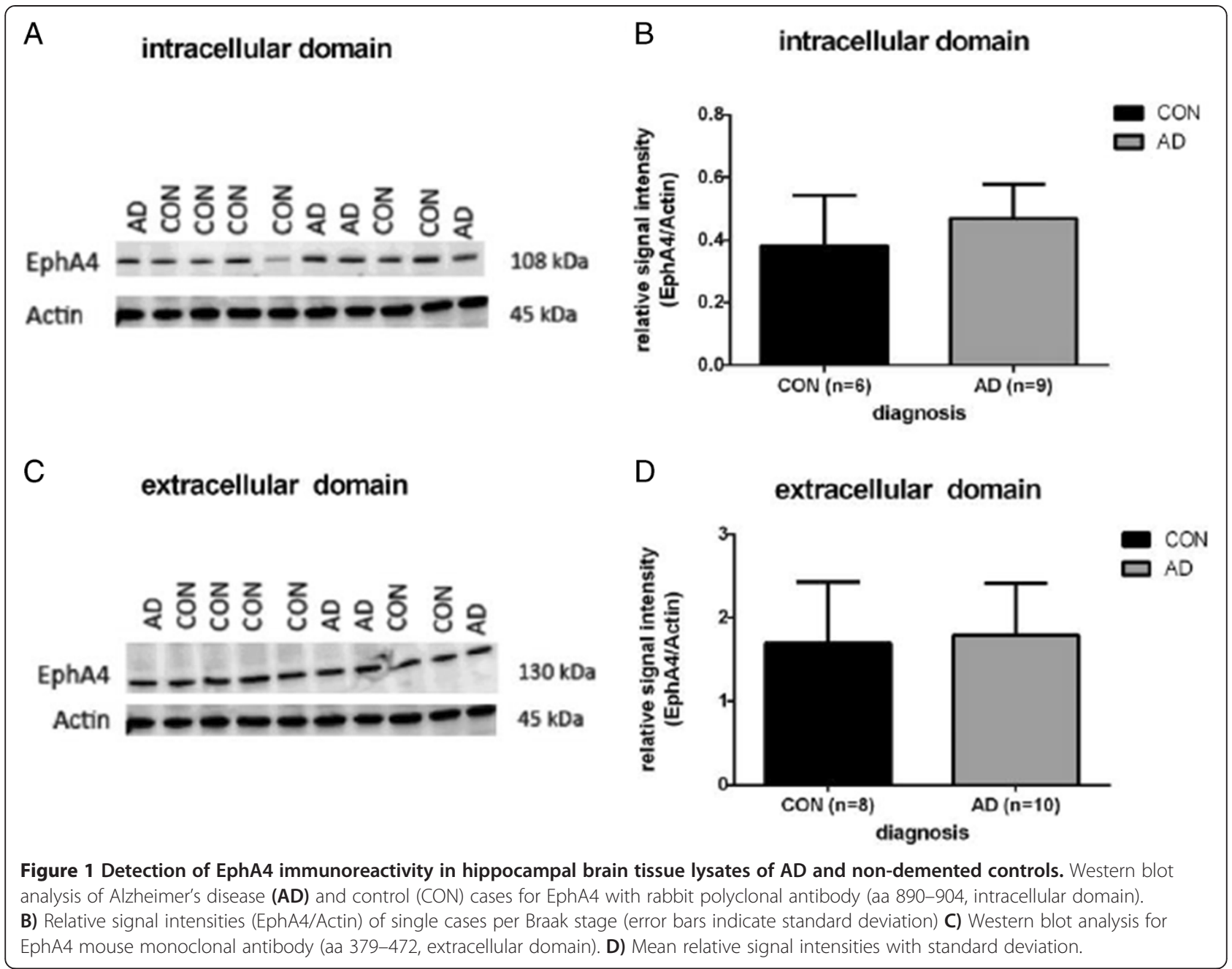

HRP rabbit/mouse antibody for $30 \mathrm{~min})$. Subsequently, colour was developed using 3,3-diaminobenzidine (EVDAB; DAKO) as chromogen. Sections were counterstained with hematoxylin and mounted using Quick-D mounting medium (Klinipath B.V., Duiven, The Netherlands) (Figures 2 and 3). Between all incubation steps, sections were washed extensively with phosphate buffered saline (PBS, pH 7.4), which also served as a negative control by omission of primary antibodies.

\section{Double immunohistochemistry: EphA4 with either $A \beta$ or pTau}

To determine the co-localization of EphA4 with amyloid beta and phosphorylated tau double immunohistochemistry was performed. Hippocampal brain slices were pre-incubated with serum-free protein blocking (SFPB, DAKO) for $10 \mathrm{~min}$ and subsequently incubated with mouse monoclonal anti-EphA4 [25-27] (1:200, IgG1, BD Transduction Laboratories $^{\mathrm{TM}}$ ) for 1 hour. After washing with PBS, slices were incubated with EnVision solution (goat anti-rabbit HRP, undiluted, DAKO) for 30 minutes.
Colour was developed using DAB as chromogen. Sections were treated with $10 \mathrm{mM}$ sodium citrate buffer $\mathrm{pH} 6.0$ heated at 180 Watt for 10 minutes. After pre-incubation with SFPB for 10 minutes, sections were incubated with anti-A $\beta$ VU 1-17 antibody [29] (1:1000, IgG2a, VU medical center) or anti-pTau AT8 antibody (dilution 1:200, IgG1) for 1 hour. Sections were washed with PBS and incubated with either GaMIgG2a ${ }^{\text {HRP }}$ (dilution 1:100, Southern Biotech) for the detection of VU $1-17$ or GoMIgG1 ${ }^{\mathrm{AF}}$ (dilution 1:100, Southern Biotech) for the detection of AT8 for 1 hour at RT. Colour was developed using Liquid Permanent Red (LPR, DAKO) as chromogen. Slices were counterstained with hematoxylin and mounted using Aquamount (BDH Laboratories Supplies). The Nuance ${ }^{\text {TM }}$ spectral imaging system (CRi, Woburn, MA) was used for the analysis of double stained specimens. Spectral imaging data cubes were taken from 460-660 $\mathrm{nm}$ at $10 \mathrm{~nm}$ intervals and analyzed with the Nuance ${ }^{\mathrm{TM}}$ software. Spectral libraries of single-brown (DAB), single-red (LPR) and hematoxylin were obtained from control slides. The resulting library was applied to the double stained slides 


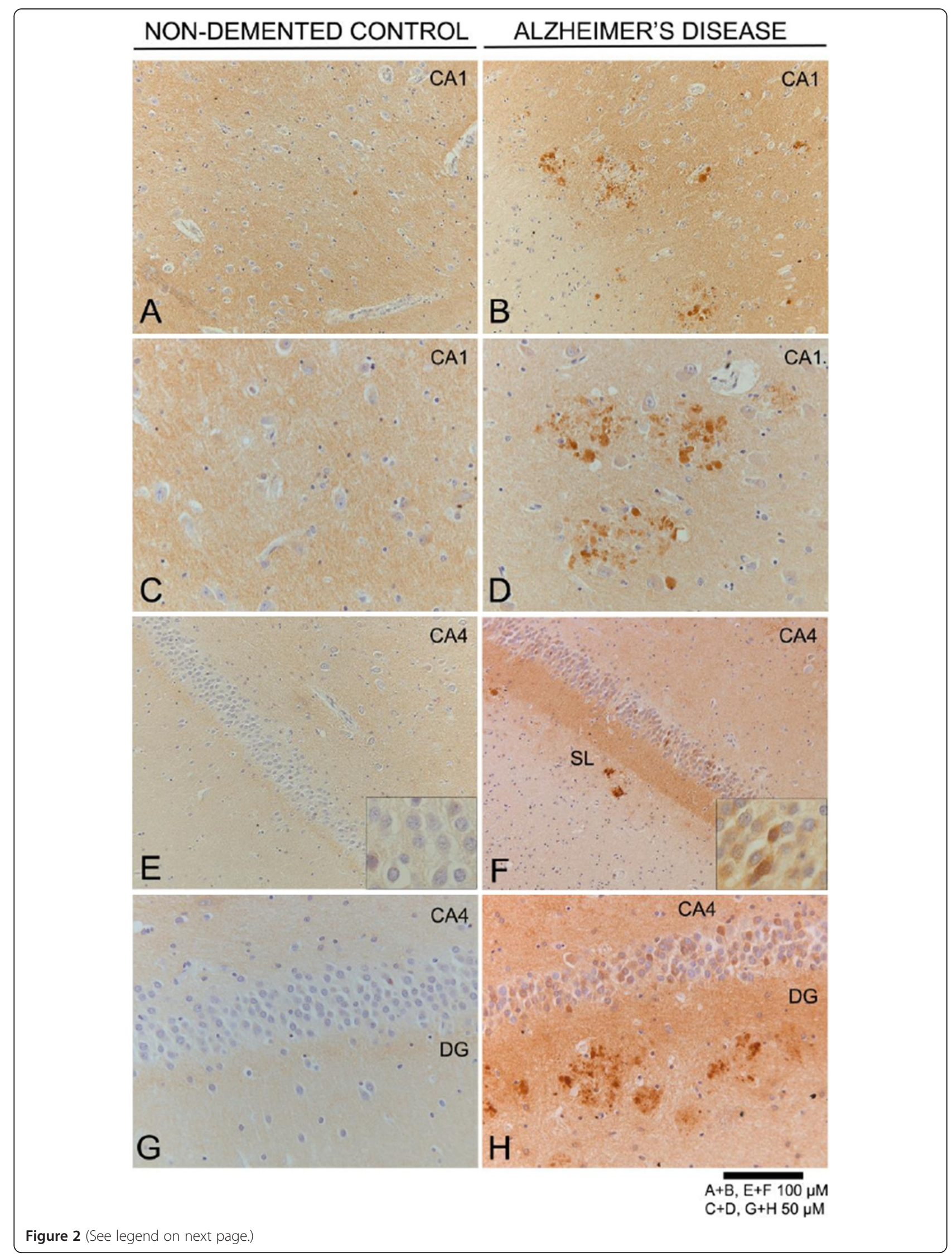


(See figure on previous page.)

Figure 2 Detection of EphA4 immunoreactivity in non-demented controls and AD hippocampus. Immunohistochemical detection of EphA4 (DAB i.e. brown colour) in the CA1 region of A) non-demented control (Braak 2 O, \#7 in Table 1) and B) AD (Braak 6 C, case \#32 in Table 1) and with $2 x$ higher magnification $\mathbf{C}$ ) and $\mathbf{D}$ ) respectively. EphA4 immunoreactivity in the dentate gyrus (DG) and stratum lacunosum (SL) in E) control (Braak 1 B, \#5 in Table 1) and F) AD (Braak 6 C, \#30 in Table 1) with 2x higher magnification in $\mathbf{G}$ ) and $\mathbf{H}$ ) respectively. Inserts in E) and F) are $2 x$ higher magnifications of neurons of the granular layer (GL). The first panel A-D shows the CA1 region, whereas the second panel $\mathbf{E}-\mathbf{H}$ are images from the CA4 and granular layer. All sections were counterstained with hematoxylin. Scale bar $100 \mu m$ for $A+B, E+F$ and $50 \mu m$ for $C+D, G+H$.

and the different reaction products were then spectrally unmixed into individual black-and-white images, representing the localization of each of the reaction products, and reverted to fluorescence-like images composed of pseudo-colours using the Nuance ${ }^{\mathrm{TM}}$ software (Figure 4).

\section{Analysis and quantification of EphA4 immunostaining}

Presence of EphA4 in hippocampal subregions CA1 and subiculum were analysed in sections immunostained with EphA4 (BD Transduction Laboratories) by an observer blinded to the clinical and pathological diagnosis. Microscopic fields $(n=4$, magnification 100x) were analysed (Table 2). The number of EphA4 depositions was scored as follows: 0 , none; 1 , one to ten depositions; 2 , eleven to twenty depositions; 3 , more than twenty. The presence of $\mathrm{A} \beta$ plaques (plaque severity) was quantified in a similar manner $(0$, none; 1 , occasional plaque; 2, several plaques scattered throughout the field; 3 , abundant presence of plaques). The presence of pTautangles was scored: 0 , none; 1 , mild (occasional immunoreactivity); 2 , moderate (scattered throughout the field); 3 , severe (abundant presence of plaques). Additional file 3 : Figure S7 shows examples for scoring of EphA4, A $\beta$ and pTau immunoreactivity.

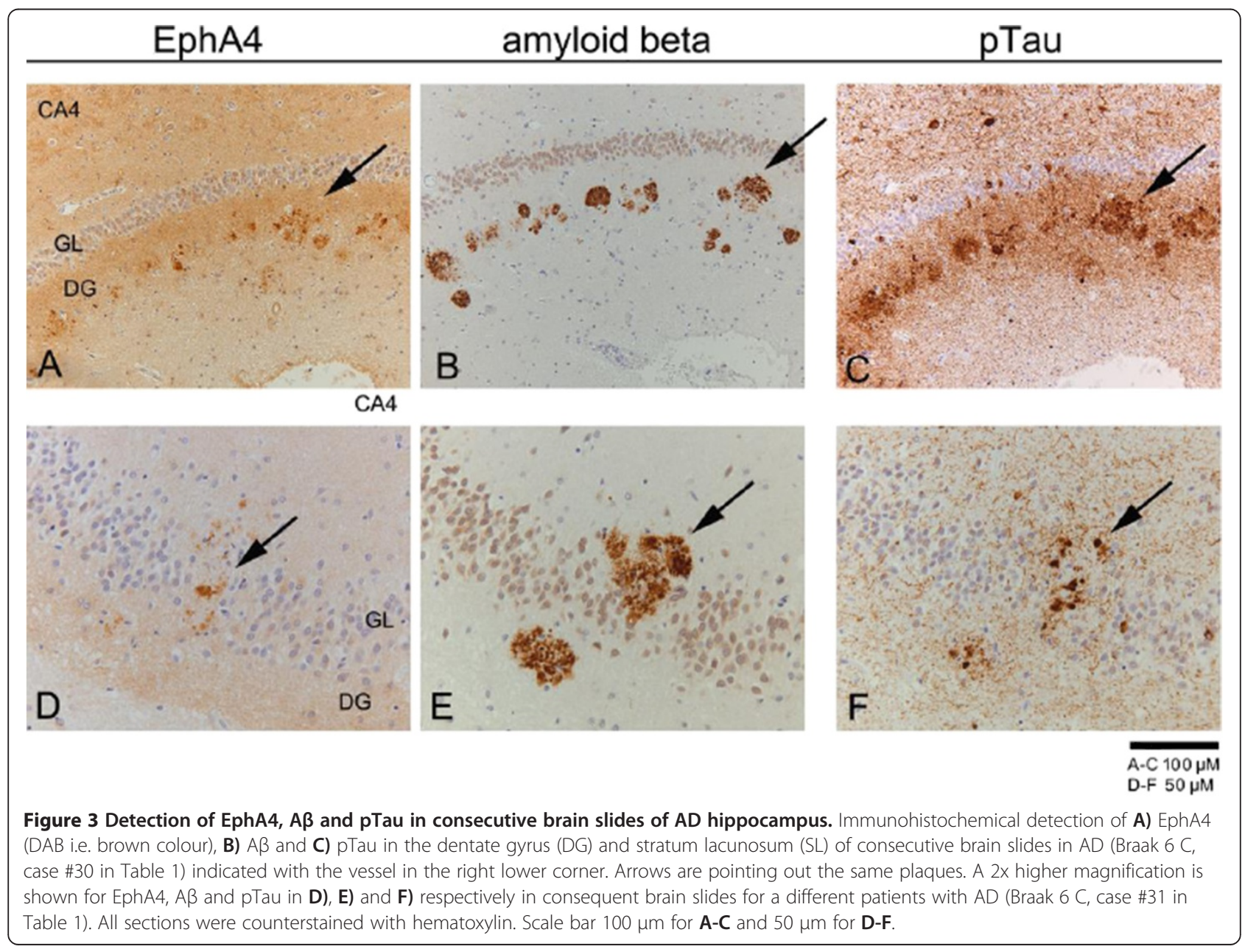




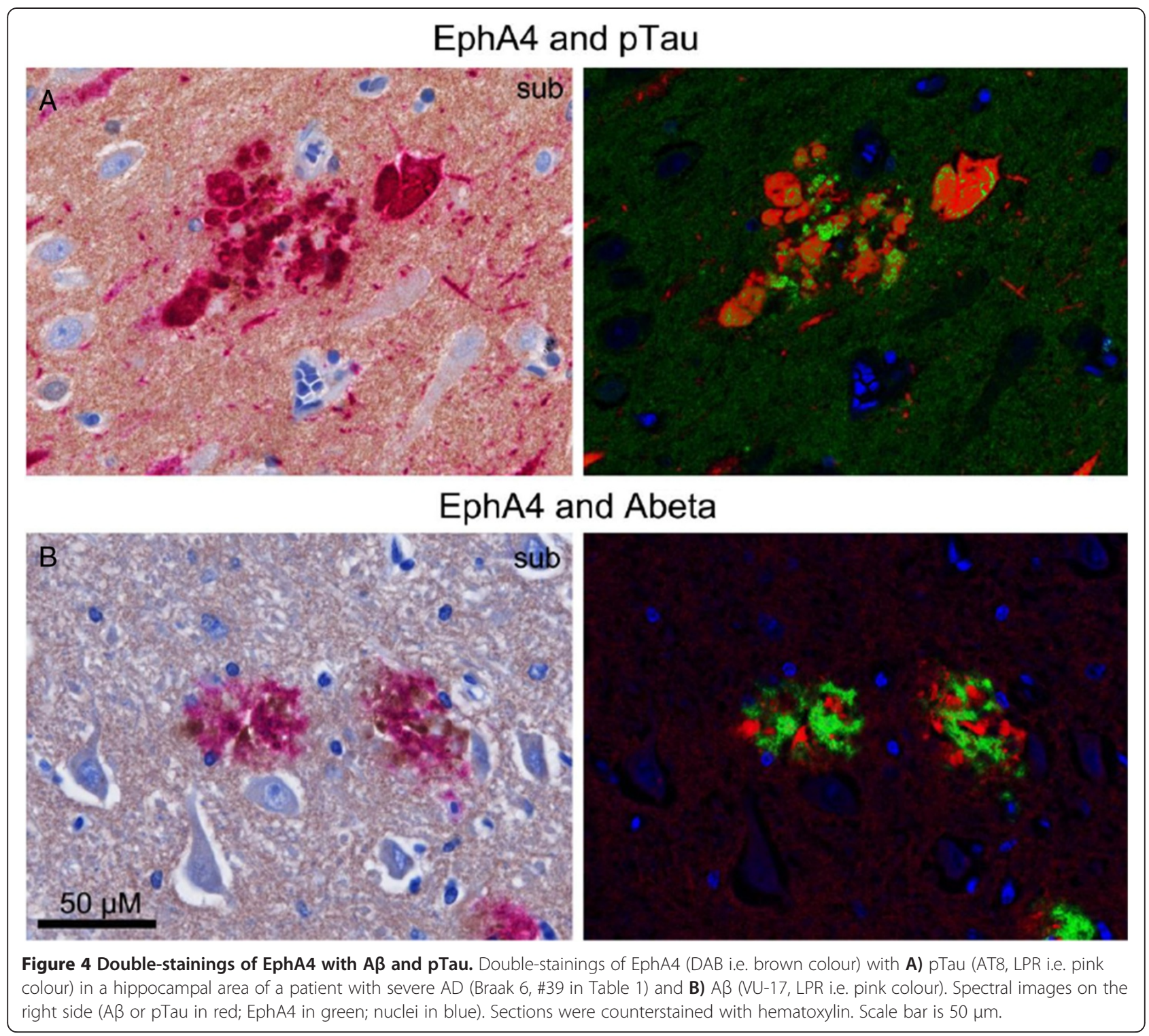

\section{Statistical analysis}

Pairwise correlation analyses for non-parametric ordinal data were conducted using IBM SPSS Statistics 22.0 (Figure 5A-D). Spearman's correlation coefficients and Kendall's tau coefficients were calculated. Correlations were considered significant in the $95 \%$ confidence interval when $\mathrm{p}<0.05$ (Table 3).

\section{Results}

In the present study we report on the involvement of EphA4 in human AD pathology. We determined the levels of EphA4 in hippocampal brain tissue of AD and non-demented control patients and found an association with the two hallmarks of AD (pTau and Abeta) using immunohistochemical analysis.
Expression levels of EphA4 in the human hippocampus at different stages of $A D$

In order to investigate protein levels of EphA4 we analysed frozen hippocampal brain tissue lysates of AD patients $(n=18)$ and non-demented controls $(n=11)$ by Western Blotting. Using two different EphA4 antibodies i.e. a rabbit polyclonal antibody raised against the intracellular part of the EphA4 and a mouse monoclonal antibody to detect the extracellular domain of EphA4. The rabbit antibody detected a protein (fragment) of approx. $108 \mathrm{kDa}$ whereas the mouse antibody showed a band that correlates to a molecular weight of $130 \mathrm{kDa}$ (Figure $1 \mathrm{~A}, \mathrm{C}$ ). Actin was used as a loading control (molecular weight is $\sim 45 \mathrm{kDa}$ ) and to determine relative signal intensities for EphA4 (Figure 1B,D). We observed no significant difference in the levels of the intracellular 
Table 2 Scoring of EphA4, A $\beta$ plaques, pTau plaques and pTau tangles

\begin{tabular}{|c|c|c|c|c|c|c|}
\hline Case no. & Braak & Braak (amyloid) & EPHA4 plaques & pTau plaques & pTau tangles & $\mathrm{A} \beta$ plaques \\
\hline 1 & 1 & C & + & ++ & + & ++ \\
\hline 2 & 1 & B & 0 & 0 & 0 & +++ \\
\hline 3 & 1 & B & + & 0 & + & +++ \\
\hline 4 & 1 & B & + & 0 & 0 & + \\
\hline 5 & 1 & B & 0 & 0 & + & ++ \\
\hline 6 & 1 & 0 & 0 & 0 & 0 & 0 \\
\hline 7 & 2 & 0 & + & 0 & ++ & 0 \\
\hline 8 & 2 & 0 & 0 & 0 & +++ & 0 \\
\hline 9 & 2 & 0 & +++ & 0 & 0 & 0 \\
\hline 10 & 2 & B & + & +++ & + & +++ \\
\hline 11 & 2 & B & ++ & + & ++ & ++ \\
\hline 12 & 2 & 0 & + & 0 & ++ & + \\
\hline 13 & 3 & C & +++ & +++ & +++ & +++ \\
\hline 14 & 3 & B & 0 & + & + & +++ \\
\hline 15 & 3 & A & + & 0 & ++ & + \\
\hline 16 & 3 & B & + & ++ & ++ & + \\
\hline 17 & 3 & C & + & ++ & +++ & +++ \\
\hline 18 & 3 & C & + & 0 & + & +++ \\
\hline 19 & 4 & C & +++ & ++ & +++ & +++ \\
\hline 20 & 4 & C & + & +++ & ++ & ++ \\
\hline 21 & 4 & C & ++ & 0 & ++ & +++ \\
\hline 22 & 4 & C & + & + & +++ & +++ \\
\hline 23 & 4 & C & +++ & + & +++ & +++ \\
\hline 24 & 5 & C & +++ & ++ & +++ & +++ \\
\hline 25 & 5 & C & +++ & +++ & +++ & ++ \\
\hline 26 & 5 & C & +++ & +++ & ++ & +++ \\
\hline 27 & 5 & C & + & +++ & ++ & +++ \\
\hline 28 & 5 & C & + & 0 & +++ & +++ \\
\hline 29 & 5 & $B$ & +++ & +++ & + & +++ \\
\hline 30 & 6 & C & +++ & +++ & +++ & +++ \\
\hline 31 & 6 & C & +++ & + & +++ & +++ \\
\hline 32 & 6 & C & +++ & ++ & +++ & +++ \\
\hline 33 & 6 & C & 0 & +++ & +++ & +++ \\
\hline 34 & 6 & C & +++ & +++ & +++ & +++ \\
\hline 35 & 6 & C & + & +++ & ++ & +++ \\
\hline
\end{tabular}

and extracellular domain of EphA4 between AD cases and controls (Figure 1). We analysed the levels of EphA4 at different stages of $\mathrm{AD}$ and found no significant differences (results not shown). Since EphA4 is an integral membrane protein we analysed whole tissue lysates (containing soluble and insoluble fraction). No difference in average levels was observed between $\mathrm{AD}$ and control hippocampus (see Additional file 1: Figure S6). In conclusion we observed no differences in EphA4 levels in hippocampal tissue at different stages of AD. Recombinant proteins EphA1, EphA4 and EphB2 were used to prove specificity of the rabbit polyclonal EphA4 antibody. The mouse anti-EphA4 antibody detects EphA4 at aa 379472. This sequence is not present in recombinant EphA4 which represents a shorter fragment of EphA4. Therefore mouse anti-EphA4 antibody is not able to detect recombinant EphA4 (Additional file 2: Figure S8). No bands were observed for recombinant EphA1 and EphB2 whereas a band for recombinant EphA4 was detected at $72 \mathrm{kDa}$ for all three concentrations (1:50, 

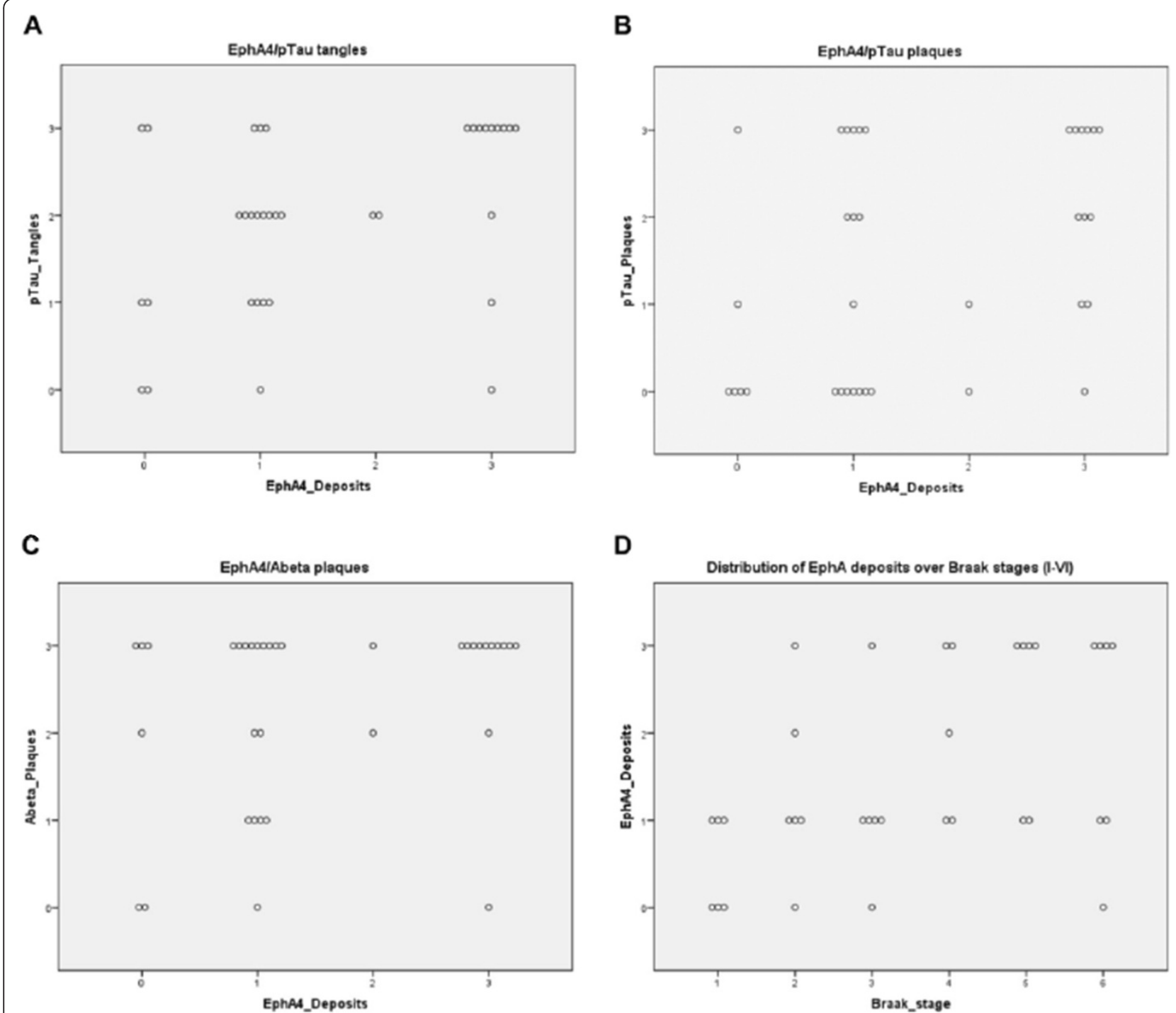

Figure 5 Pairwise correlation analysis of EphA4, pTau plaques and tangles and A $\boldsymbol{\beta}$ plaques. Correlation of EphA4 deposits with A) pTau tangles, B) pTau plaques and C) Abeta plaques. D) Distribution of EphA4 deposits over Braak stages (I-VI). E) Table with the corresponding correlation coefficients (Spearman's rho and Kendall's tau) as well as p-values. Correlations are considered significant when $p<.05$.

1:100, 1:200). This suggests highly specific binding of the rabbit EphA4 antibody.

\section{EphA4 localization in patients with Alzheimer's disease}

Since we observed no difference in the levels of EphA4 we wondered whether the localisation of EphA4 might change in $\mathrm{AD}$ versus non-demented controls. The mouse monoclonal antibody directed at the extracellular (c-terminal) domain of EphA4 was used for detection of the protein. In non-demented control cases (without $\mathrm{AD}$ pathology), a low intense immune-reactive signal of EphA4 was detected in the parenchyma. The

Table 3 Correlation coefficients for pairwise comparisons of EphA4 with the hallmarks of AD and Braak stage (according to Figure 5)

\begin{tabular}{lllll}
\hline & & Kendall's tau $[\boldsymbol{\tau}]$ & Spearman's rho $\left[\mathbf{r}_{\mathbf{s}}\right]$ & significance $[\mathbf{p}<.05]$ \\
\hline A & EphA4/pTau tangles & $.393, p=.007^{*}$ & $.420, p=.011^{*}$ & significant \\
B & EphA4/pTau plaques & $.329, p=.025^{*}$ & $.378, p=.023^{*}$ & significant \\
C & EphA4/Abeta plaques & $.259, \mathrm{p}=.084$ & $.282, \mathrm{p}=-096$ & not significant \\
$\mathrm{D}$ & EphA4/Braak stage & $.421, \mathrm{p}=.003^{*}$ & $.486, \mathrm{p}=.003^{*}$ & significant \\
\hline
\end{tabular}


signal was evenly distributed throughout the hippocampus (Figure 2A,C). In contrast, in $\mathrm{AD}$ cases EphA4 immuno-reactivity was observed in plaque-like structures (Figure 2B,D). Interestingly, EphA4 immuno staining of these plaque-like structures was observed more frequently in the higher Braak stages (V and VI), but some of the samples representing low stages (three in Braak I and 5 in Braak II) showed the same pattern. The EphA4-positive plaque-like structures showed considerable variations in shape and size $(5-50 \mu \mathrm{m})$ and were detected in all subregions of the hippocampus.

Intense EphA4 immunostaining was observed in the cytoplasm of granular neurons in the dentate gyrus of $\mathrm{AD}$ but not in the dentate gyrus of non-demented control cases (Figure 2E,F). In non-demented controls, EphA4 immunoreactivity was found as a fine punctate line in the plexiform layers of the dentate gyrus (Figure 2G). In AD patients, EphA4-positivity was frequently decorating the dentate gyrus showing a brown band in the perforant path target zone of the outer molecular layer of the dentate gyrus, forming a frame around the granular layer (Figure 2F,H). This staining pattern was not observed in control cases. EphA4-positivity was not restricted to specific subareas of the hippocampus but was also found in and around neurons of the granular layer and in the stratum lacunosum. The presence of EphA4 immunoreactive plaque-like deposits in the CA regions of the hippocampus and entorhinal cortex in combination with the intense EphA4 immunostaining of the molecular layer was most prominent in demented individuals. Interestingly, EphA4 immunoreactive plaques were also observed in the hippocampi of non-demented controls (Braak II, see scoring Table 2) which points towards an early involvement of EphA4 in AD pathology.

\section{EphA4 partially co-localizes with pTau and neuritic A $\beta$ plaques}

The detection of EphA4 immunoreactivity plaque-like structures suggests a co-localization with neuritic plaques. In order to investigate the co-occurrence of EphA4 with neuritic plaques, adjacent brain tissue sections were stained for EphA4, pTau and A $\beta$ (Figure 3). Consecutive brain slices were stained for $A \beta$, pTau and EphA4 and partial co-localization in the hippocampus of AD patients was revealed.

In order to corroborate the possible co-localization of EphA4 with either A $\beta$ or pTau, double-immunostaining was performed. We found confirmation of partial co-localization of EphA4 with pTau and $A \beta$-immunoreactive plaques in AD patients (Figure 4A). EphA4-immunoreactivity was characteristically found at the ends of dystrophic neurites around central A $\beta$ plaques (Figure $4 \mathrm{~B}$ ). EphA4 immunoreactivity is less intense compared to that of $A \beta$ or pTau which could reflect differences in the antigen binding properties of the different antibodies. So EphA4 localization is clearly linked to the deposition of both $\mathrm{A} \beta$ and $\mathrm{pTau}$.

\section{EphA4 depositions in the hippocampus of AD patients at different Braak stages}

In order to investigate whether the number of EphA4 depositions increases with Braak stage and correlates with the hallmarks of $\mathrm{AD}$, immunoreactivity for EphA4 was analysed in a new second cohort representing all Braak stages (I-VI) (Table 2). Adjacent tissue sections were stained and scored for EphA4 depositions, A $\beta$ plaques, pTau-positive plaques and pTau tangles (see Additional file 3: Figure S7 for scoring example).

To examine the relationship between EphA4 and the hallmarks of AD, Spearman's correlation $\left[\mathrm{r}_{\mathrm{s}}\right.$ ] and Kendall's correlation $[\tau]$ analyses for non-parametric ordinal data were performed (Table 3). Spearman's correlation coefficients are used for correlation analyses of non-parametric data sets. Kendall's correlation coefficients are preferred for small sample sizes with a large number of tied ranks. The analyses revealed a significant correlation between EphA4 positive depositions and pTau-positive tangles (Figure 5A; $\mathrm{r}_{\mathrm{s}}=.420, \mathrm{p}<.05 ; \mathrm{\tau}=.393, \mathrm{p}<.05$ ) and between EphA4 and pTau-positive plaques (Figure 5B; $\mathrm{r}_{\mathrm{s}}=.378$, $\mathrm{p}<.05 ; \mathrm{\tau}=-.329, \mathrm{p}<.05)$. No significant correlation was found between EphA4-load and $\mathrm{A} \beta$ plaques (Figure $5 \mathrm{C}$; $\mathrm{r}_{\mathrm{s}}=.282$, ns; $\mathrm{\tau}=.259, \mathrm{~ns}$ ). However, the correlation between EphA4 deposits and Braak stage was evident (Figure 5D; $\left.\mathrm{r}_{\mathrm{s}}=.486, \mathrm{p}<.05 ; \mathrm{\tau}=.421, \mathrm{p}<.05\right)$. Table 3 summarizes the obtained correlation coefficients and $\mathrm{p}$-values.

\section{Discussion}

Synaptic loss is one of the major pathological hallmarks of AD. This is considered to be an early event in the pathogenesis of the disease. Synaptic failure correlates with cognitive decline and is observed in patients with mild cognitive impairment (MCI) and incipient AD [3]. The molecular mechanism of synaptic dysfunction in $\mathrm{AD}$ remains elusive.

The tyrosine kinase receptor EphA4 is essential for synaptic function as it is involved in dendritic spine morphogenesis, synapse formation and maturation [5]. EphA4 is highly expressed in the adult hippocampus, where it is known to play a role in adult synaptic plasticity and learning $[16,30]$. Of all cortical areas, the hippocampus appears to be most severely affected by the loss of synaptic proteins in $\mathrm{AD}$ (44 to 55\%) [31]. Interestingly, analysis of synaptoneurosomes from AD patients revealed a 2-fold increase in EphA4 mRNA [32], suggesting a role in synaptotoxicity for EphA4 Therefore we investigated EphA4 expression and localization in the hippocampus of patients at various Braak stages. 
In this study the total EphA4 protein levels were similar in $\mathrm{AD}$ patients compared to control cases. However, immunohistochemical localization of EphA4 revealed an altered distribution in AD compared to control hippocampus. EphA4 partially co-localized with neuritic amyloid beta plaques. An aberrant function of EphA4 might be the underlying cause for the altered distribution of EphA4 in different Braak stages. Thus, EphA4 could be contributing to synaptic dysfunction which is considered an early event in AD.

So far, reports about changes in EphA4 protein levels in $\mathrm{AD}$ hippocampus have been contradictory. Simón et al. reported a reduction of EphA4 (20\%) in hippocampal tissue of three patients with very mild cognitive deficits (Braak stage II and III) compared to three control subjects [33]. Matsui et al. showed that total protein levels of EphA4 in AD brains were not altered compared to controls. Furthermore they reported a decrease of membrane associated EphA4 (intracellular domain) in frontal lobes of AD cases while the amount of fulllength EphA4 was unchanged [34]. In the present study we investigated a large cohort $(n=35)$ covering all Braak stages using two different antibodies directed at different epitopes of EphA4. EphA4 fragments with different molecular weights (108 kDa vs. $130 \mathrm{kDa})$ were detected. This discrepancy can be explained by the different binding sites of the used antibodies. Overall, we were not able to detect changes in EphA4 protein levels between control and AD groups and between different Braak stages when we used Western Blot analysis.

In contrast, at the immunohistochemical level we observed differences in the staining patterns of EphA4 when comparing control and AD cases. We showed that depositions of the EphA4 protein kinase are present in all subareas of the hippocampus in AD patients. The number of EphA4 deposits increases with Braak stage and those deposits partially co-localize with neuritic plaques and tangles. Moreover, EphA4 immunoreactive plaques are already present in Braak stage II which points towards an involvement of EphA4 in the early stages of $\mathrm{AD}$ pathology. The increased occurrence of EphA4 deposits with AD pathology in the absence of changes in total EphA4 protein levels indicate an altered distribution of EphA4. The important role of EphA4 in synaptic dysfunction, an early event in $\mathrm{AD}$, has been reported [35]. Pathological changes happen in the brain even decades before the first clinical symptoms emerge. Therefore, it is likely that the aberrant EphA4 staining in part of the control cases (Braak stage II and III) poses an early event in $\mathrm{AD}$ pathogenesis and is therefore specific.

Mostly, investigations into $\mathrm{AD}$ related synaptic changes have focused on the toxic effects of $A \beta$. Like the amyloid precursor protein (APP), EphA4 is a substrate of $\gamma$ - secretase [34]. The EphA4 intracellular domain (EICD) that remains after cleavage is known to enhance the formation of dendritic spines via activation of the Rac signaling pathway [34]. It has been suggested that aberrant $\gamma$-secretase activity followed by hindered cleavage of EphA4 results in reduced formation of dendritic spines and may be the major cause of synaptic failure in $\mathrm{AD}$ [36]. In addition, reduced EphA4 levels have been reported in whole-cell lysates of $\mathrm{hA} \beta \mathrm{PP}_{\text {swe-ind }}$ mice and Tg2576 mice compared to non-transgenic mice [33]. Those $\mathrm{hA} \beta \mathrm{PP}_{\text {swe-ind }}$ mice show amyloid-related pathology but no accumulation of pTau in tangles. These data strengthen the possible link between $\mathrm{A} \beta$ and aberrant EphA4 signaling.

The emerging view is that toxic amyloid- $\beta$ oligomers $(\mathrm{A} \beta \mathrm{Os})$ are an important pathological factor in early neurodegenerative events in $\mathrm{AD}$ [37]. Two major forms of $\mathrm{A} \beta$ coexist in the brain: a shorter form with 40 amino acid residues and a longer form with 42 amino acids. The longer form is extremely toxic and can self-aggregate to form oligomers (amyloid beta oligomers, A $\beta O s$ ) [2]. Those oligomers accumulate into $A \beta$ deposits in patients with $A D$. It has been reported recently that activation of the Abelson non-receptor tyrosine kinase c-Abl, a kinase downstream of EphA4, mediates synaptic loss and long term potentiation in dendritic spines of cultured rat hippocampal neurons. The co-localization of c-Abl with amyloid plaques, neurofibrillary tangles and granulovacuolar degeneration in the hippocamups and entorhinal cortex of $\mathrm{AD}$ patients has been reported in 2009 by Jing et al. [38]. AßOs activate the c-Abl kinase and thereby induce synaptic loss [21]. Concomitantly, EphA4 tyrosine phosphorylation is increased in these cultured neurons and in synaptoneurosomes exposed to A $\beta O s$. EphA4/c-Abl activation is a key-signaling event mediating the synaptic damage induced by AßOs. EphA4/c-Abl signaling could hence be a relevant pathway involved in the early cognitive decline observed in AD patients [21].

In human brain, a stronger relation between EphA4 and pTau positive plaques exists. In $\mathrm{AD}, \mathrm{c}-\mathrm{Abl}$ is detected in neurofibrillary tangles [38] and phosphorylates tau directly [39] and through activation of Cdk5 [40]. In the present study, we show a significant correlation between EphA4 positive depositions and pTau-positive plaques and an almost significant correlation with pTau-positive tangles in AD. Also Matsui et al. previously reported a correlation between the intracellular domain of EphA4 (EICD) and tau phosphorylation, although the correlation did not reach statistical significance. In contrast, the level of EICD did not correlate with the level of $A \beta$ [34].

Tau may be involved in synaptic dysfunction in dementia [36], strengthening the association with EphA4 [5]. A key question remains whether the association with EphA4 is causally related to tau phosphorylation and 
aggregation. Either aberrant EphA4 signaling promotes phosphorylation of tau contributing to the formation of neurofibrillary tangles and neuronal dystrophy, or on the contrary, aggregating tau affects the subcellular distribution of key proteins involved in synaptic function such as the EphA4 receptor. So far several groups have reported that Eph/ephrin signaling up-regulates tau expression and phosphorylation [41,35]. When EphA4 is activated by ephrin A1 it recruits and phosphorylates cyclin-dependent kinase 5 (CDK5) [35]. CDK5 is a tau kinase and is increased in AD brain [42]. Increased CDK5 immunoreactivity is observed in pretangle neurons supporting its involvement in early stages of AD pathogenesis [43]. The significance of a dysregulation of CDK5 by EphA4 in pathological conditions remains elusive.

In our study, EphA4 depositions partially co-localize ( $\sim 30 \%)$ with neuritic $A \beta$ plaques in human hippocampal brain tissue. Manczak et al. recently reported a physical interaction between $\mathrm{A} \beta$ and phosphorylated Tau [44]. They found an abnormal interaction of oligomeric $A \beta$ with pTau in neurons of post mortem brains from AD patients. This interaction may be involved in neuronal and synaptic damage, leading to cognitive decline in incipient AD patients [44]. Those findings may explain that EphA4 is related to both $\mathrm{A} \beta$ and pTau and suggests an underlying common pathway. Further studies are necessary to shine light on the relation of those proteins and the significance in the progression of $\mathrm{AD}$.

Four recent large late-onset AD (LOAD) genome-wide association studies (GWAS) have identified EPHA1 as a genetic factor. Like EphA4, EphA1 is suggested to be important for synaptic function $[12,45]$. This supports the emerging evidence that the Eph receptors and their ligands, the ephrins, are involved in aberrant synaptic functions associated with cognitive impairment in $\mathrm{AD}$ [5]. In this study we show that EphA4 co-localizes with neuritic plaques in human brain tissue of patients with AD. EphA4 is associated with both $A \beta$ and pTau. The altered distribution of EphA4 in AD hippocampus may reflect a decreased function of EphA4, which is likely to contribute to synaptic dysfunction that occurs in the early stages of AD. Pathological changes happen decades before the first clinical symptoms emerge explaining EphA4positivity in early Braak stages (II and III, see Table 2).

\section{Additional files}

Additional file 1: Figure S6. Example of EphA4 detection in whole tissue lysates by Western blot analysis. A) Western blots with whole tissue lysates of hippocampi of all Braak stages (0-VI). B) Box blot analysis of Western Blots for EphA4. Signal intensity for Braak 0-III and Braak IV-VI. Specificity $\mathrm{p}<0.05$.

Additional file 2: Figure S8. Western Blot analysis of specificity of EphA4 (rabbit) antibody against recombinant EphA1 and EphB2. The rabbit polyclonal EphA4 antibody is specific for EphA4 (band at $72 \mathrm{kDa}$ ). No bands are observed for recombinant EphA1 and EphB2.

Additional file 3: Figure S7. Example of scoring for quantification of EphA4, pTau and A $\beta$. For explanation of scoring see material and methods and Table 2.

\section{Competing interests}

The authors have no conflicts of interests to declare.

\section{Authors' contributions}

AFNR coordinated the study and was responsible for writing the manuscript. AFNR and JJMH designed and performed experiments. AJMR was responsible for the autopsy material and neuropathological evaluation. SMvdV, AJMR, WvdF, PS and JJMH made intellectual contributions to experimental design and discussion. All authors read and approved the final manuscript.

\section{Acknowledgements}

We thank the Netherlands Brain Bank for supplying human brain tissue, Elise van Haastert, David Hondius, Marlies Jacobs, Marloes van Roijen and Sjoukje Eijbersen for their assistance in the laboratory and Riet Hilhorst for critical reading of the manuscript. We thank Robert Veerhuis for providing the VU17 antibody.

\section{Funding}

This study was financially supported by Stichting Zabawas and Agentschap NL. Research at the VUmc Alzheimer center and the Department of Pathology is part of the neurodegeneration research program of the Neuroscience Campus Amsterdam. The VUmc Alzheimer center is supported by Alzheimer Nederland and Stichting VUmc fonds.

Received: 28 May 2014 Accepted: 26 June 2014 Published: 16 July 2014

\section{References}

1. Selkoe DJ (2001) Alzheimer's disease: genes, proteins, and therapy. Physiol Rev 81:741-766

2. DeKosky ST, Scheff SW (1990) Synapse loss in frontal cortex biopsies in Alzheimer's disease: correlation with cognitive severity. Ann Neurol 27:457-464, doi:10.1002/ana.410270502

3. Arendt T (2009) Synaptic degeneration in Alzheimer's disease. Acta Neuropathol 118:167-179, doi:10.1007/s00401-009-0536-X

4. Terry RD, Masliah E, Salmon DP, Butters N, DeTeresa R, Hill R, Hansen LA, Katzman R (1991) Physical basis of cognitive alterations in Alzheimer's disease: synapse loss is the major correlate of cognitive impairment. Ann Neurol 30:572-580, doi:10.1002/ana.410300410

5. Chen Y, Fu AKY, Ip NY (2012) Eph receptors at synapses: Implications in neurodegenerative diseases. Cell Signal 24:606-611, doi:10.1016/j. cellsig.2011.11.016

6. Chen Y, Fu AKY, Ip NY (2008) Bidirectional signaling of ErbB and Eph receptors at synapses. Neuron Glia Biol 4:211-221, doi:10.1017/ S1740925X09990287

7. Pasquale EB (2008) Eph-Ephrin Bidirectional Signaling in Physiology and Disease. Cell 133:38-52, doi:10.1016/j.cell.2008.03.011

8. Castellani V, Yue Y, Gao PP, Zhou R, Bolz J (1998) Dual action of a ligand for Eph receptor tyrosine kinases on specific populations of axons during the development of cortical circuits. J Neurosci 18:4663-4672

9. Dottori M, Hartley L, Galea M, Paxinos G, Polizzotto M, Kilpatrick T, Bartlett PF, Murphy M, Köntgen F, Boyd AW (1998) EphA4 (Sek1) receptor tyrosine kinase is required for the development of the corticospinal tract. Proc Natl Acad Sci U S A 95:13248-13253, doi:9789074

10. Flanagan JG, Vanderhaeghen $P$ (1998) The ephrins and Eph receptors in neural development. Annu Rev Neurosci 21:309-345

11. Coulthard MG, Morgan M, Woodruff TM, Arumugam TV, Taylor SM, Carpenter TC, Lackmann M, Boyd AW (2012) Eph/ephrin signaling in injury and inflammation. Am J Pathol 181:1493-1503, doi:10.1016/j. ajpath.2012.06.043

12. Hollingworth P, Harold D, Sims R, Gerrish A, Lambert JC, Carrasquillo MM, Abraham R, Hamshere ML, Pahwa JS, Moskvina V, Dowzell K, Jones N, Stretton A, Thomas C, Richards A, Ivanov D, Widdowson C, Chapman J, 
Lovestone S, Powell J, Proitsi P, Lupton MK, Brayne C, Rubinsztein DC, Gill M, Lawlor B, Lynch A, Brown KS, Passmore PA, Craig D et al (2011) Common variants at ABCA7, MS4A6AVMS4A4E, EPHA1, CD33 and CD2AP are associated with Alzheimer's disease. Nat Genet 43:429-435, doi:10.1038/ng.803

13. Tournoij E, Koekman CA, Du VX, Roest M, Ruijtenbeek R, Moll FL, Akkerman JW (2012) Receptor contributes to granule secretion through Ephrin A4 receptor. Platelets 23:1-10, doi:10.3109/09537104.2011.645924

14. Bowden TA, Aricescu AR, Nettleship JE, Siebold C, Rahman-Huq N, Owens RJ, Stuart DI, Jones EY (2009) Structural Plasticity of Eph Receptor A4 Facilitates Cross-Class Ephrin Signaling. Structure 17:1386-1397, doi:10.1016/ j.str.2009.07.018

15. Tremblay M-E, Riad M, Bouvier D, Murai KK, Pasquale EB, Descarries L, Doucet G (2007) Localization of EphA4 in axon terminals and dendritic spines of adult rat hippocampus. J Comp Neurol 501:691-702, doi:10.1002/cne

16. Bouvier D, Corera AT, Tremblay M-E, Riad M, Chagnon M, Murai KK, Pasquale EB, Fon EA, Doucet G (2008) Pre-synaptic and post-synaptic localization of EphA4 and EphB2 in adult mouse forebrain. J Neurochem 106:682-695, doi:10.1111/j.1471-4159.2008.05416.x

17. Yamaguchi Y, Pasquale EB (2004) Eph receptors in the adult brain. Curr Opin Neurobiol 14:288-296, doi:10.1016/j.conb.2004.04.003

18. Murai KK, Nguyen LN, Irie F, Yamaguchi Y, Pasquale EB (2003) Control of hippocampal dendritic spine morphology through ephrin-A3/EphA4 signaling. Nat Neurosci 6:153-160, doi:10.1038/nn994

19. Fu W-Y, Chen Y, Sahin M, Zhao XS, Shi L, Bikoff JB, Lai KO, Yung WH, Fu AK, Greenberg ME, Ip NY (2007) Cdk5 regulates EphA4-mediated dendritic spine retraction through an ephexin 1-dependent mechanism. Nat Neurosci 10:67-76, doi:10.1038/nn1811

20. Grunwald IC, Korte M, Adelmann G, Plueck A, Kullander K, Adams RH, Frotscher M, Bonhoeffer T, Klein R (2004) Hippocampal plasticity requires postsynaptic ephrinBs. Nat Neurosci 7:33-40, doi:10.1038/nn1164

21. MVargas L, Leal N, Estrada LD, González A1, Serrano F2, Araya K3, Gysling K3, Inestrosa NC2, Pasquale EB4, Alvarez AR1 (2014) EphA4 Activation of c-Abl Mediates Synaptic Loss and LTP Blockade Caused by Amyloid- $\beta$ Oligomers. PLoS ONE 9:e92309, doi:10.1371/journal.pone.0092309

22. Simón AM, De Maturana RL, Ricobaraza A, Escribano L, Schiapparelli L, Cuadrado-Tejedor M, Pérez-Mediavilla A, Avila J, Del Río J, Frechilla D (2009) Early changes in hippocampal Eph receptors precede the onset of memory decline in mouse models of Alzheimer's disease. J Alzheimers Dis 17:773-786, doi:10.3233/JAD-2009-1096

23. Yamaguchi H, Haga C, Hirai S, Nakazato Y, Kosaka K (1990) Distinctive, rapid, and easy labeling of diffuse plaques in the Alzheimer brains by a new methenamine silver stain. Acta Neuropathol 79:569-572, doi:10.1007/ BF00296119

24. Braak H, Alafuzoff I, Arzberger T, Kretzschmar H, Del Tredici K (2006) Staging of Alzheimer disease-associated neurofibrillary pathology using paraffin sections and immunocytochemistry. Acta Neuropathol 112:389-404, doi:10.1007/s00401-006-0127-z

25. Becker N, Gilardi-Hebenstreit P, Seitanidou T, Wilkinson D, Charnay P (1995) Characterisation of the Sek-1 receptor tyrosine kinase. FEBS Lett 368:353-357

26. Ellis C, Kasmi F, Ganju P, Walls E, Panayotou G, Reith AD (1996) A juxtamembrane autophosphorylation site in the Eph family receptor tyrosine kinase, Sek, mediates high affinity interaction with p59fyn. Oncogene 12:1727-1736

27. Gilardi-Hebenstreit $P$, Nieto MA, Frain M, Mattéi MG, Chestier A, Wilkinson DG, Charnay P (1992) An Eph-related receptor protein tyrosine kinase gene segmentally expressed in the developing mouse hindbrain. Oncogene 7:2499-2506

28. Spillantini MG, Crowther RA, Goedert M (1996) Comparison of the neurofibrillary pathology in Alzheimer's disease and familial presenile dementia with tangles. Acta Neuropathol 92:42-48, doi:10.1007/ s004010050487

29. Verwey NA, Hoozemans JJM, Korth C, van Royen MR, Prikulis I, Wouters D, Twaalfhoven HA, van Haastert ES, Schenk D, Scheltens P, Rozemuller AJ, Blankenstein MA, Veerhuis R (2013) Immunohistochemical characterization of novel monoclonal antibodies against the N-terminus of amyloid ß-peptide. Amyloid 20:179-187, doi:10.3109/13506129.2013.797389

30. Tremblay M-E, Riad M, Chierzi S, Murai KK, Pasquale EB, Doucet G (2009) Developmental course of EphA4 cellular and subcellular localization in the postnatal rat hippocampus. J Comp Neurol 512:798-813, doi:10.1002/ cne.21922
31. Scheff SW, Price DA, Dekosky ST (2007) Synaptic alterations in CA1 in mild Alzheimer disease and mild cognitive impairment. 1501-1508

32. Williams C, Shai RM, Wu Y, Hsu YH, Sitzer T, Spann B, McCleary C, Mo Y, Miller CA (2009) Transcriptome analysis of synaptoneurosomes identifies neuroplasticity genes overexpressed in incipient Alzheimer's disease. PLoS ONE 4:e4936, doi:10.1371/journal.pone.0004936

33. Inoue E, Deguchi-Tawarada M, Togawa A, Matsui C, Arita K, Katahira-Tayama S, Sato T, Yamauchi E, Oda Y, Takai Y (2009) Synaptic activity prompts gamma-secretase-mediated cleavage of EphA4 and dendritic spine formation. J Cell Biol 185:551-564, doi:10.1083/jcb.200809151

34. Matsui C, Inoue E, Kakita A, Arita K, Deguchi-Tawarada M, Togawa A, Yamada A, Takai Y, Takahashi H (2012) Involvement of the $\gamma$-secretasemediated EphA4 signaling pathway in synaptic pathogenesis of Alzheimer's disease. Brain Pathol 22:776-787, doi:10.1111/j.1750-3639.2012.00587.x

35. Peng YR, Hou ZH, Yu X (2013) The kinase activity of EphA4 mediates homeostatic scaling-down of synaptic strength via activation of Cdk5. Neuropharmacology 65:232-243, doi:10.1016/j.neuropharm.2012.10.012

36. Pooler AM, Noble W, Hanger DP (2014) Neuropharmacology Invited review A role for tau at the synapse in Alzheimer' $s$ disease pathogenesis. NP 76:1-8, doi:10.1016/j.neuropharm.2013.09.018

37. Klein WL (2013) Synaptotoxic amyloid- $\beta$ oligomers: a molecular basis for the cause, diagnosis, and treatment of Alzheimer's disease? J Alzheimer's Dis 33 (Suppl 1):S49-S65, doi:10.3233/JAD-2012-129039

38. Jing Z, Caltagarone J, Bowser R (2009) Altered subcellular distribution of c-Abl in Alzheimer's disease. J Alzheimers Dis 17:409-422, doi:10.3233/JAD2009-1062.Altered

39. Derkinderen $P$, Scales TME, Hanger DP, Leung KY, Byers HL, Ward MA, Lenz C, Price C, Bird IN, Perera T, Kellie S, Williamson R, Noble W, Van Etten RA, Leroy K, Brion JP, Reynolds CH, Anderton BH (2005) Tyrosine 394 is phosphorylated in Alzheimer's paired helical filament tau and in fetal tau with c-Abl as the candidate tyrosine kinase. J Neurosci 25:6584-6593, doi:10.1523/JNEUROSCI.1487-05.2005

40. Cancino Gl, Perez de Arce K, Castro PU, Toledo EM, von Bernhardi R, Alvarez AR (2011) C-Abl tyrosine kinase modulates tau pathology and Cdk5 phosphorylation in AD transgenic mice. Neurobiol Aging 32:1249-1261, doi:10.1016/j.neurobiolaging.2009.07.007

41. Moreno-Flores MT, Díaz-Nido J, Wandosell F, Avila J (2002) Olfactory ensheathing glia: Drivers of axonal regeneration in the central nervous system? J Biomed Biotechnol 2002:37-43, doi:10.1155/S1110724302000372

42. Shukla V, Skuntz S, Pant HC (2012) Deregulated Cdk5 Activity Is Involved in Inducing Alzheimer 's Disease. Arch Med Res 43:655-662, doi:10.1016/j. arcmed.2012.10.015

43. Morgan K (2011) The three new pathways leading to Alzheimer's disease. Neuropathol Appl Neurobiol 37:353-357, doi:10.1111/j.13652990.2011.01181.x

44. Manczak M, Reddy PH (2013) Abnormal interaction of oligomeric amyloid- $\beta$ with phosphorylated tau: implications to synaptic dysfunction and neuronal damage. J Alzheimers Dis 36:285-295, doi:10.3233/JAD-130275

45. Cissé M, Halabisky B, Harris J, Devidze N, Dubal DB, Sun B, Orr A, Lotz G, Kim DH, Hamto P, Ho K, Yu GQ, Mucke L (2011) Reversing EphB2 depletion rescues cognitive functions in Alzheimer model. Nature 469:47-52, doi:10.1038/nature09635

\section{doi:10.1186/s40478-014-0079-9}

Cite this article as: Rosenberger et al:: Altered distribution of the EphA4 kinase in hippocampal brain tissue of patients with Alzheimer's disease correlates with pathology. Acta Neuropathologica Communications $20142: 79$. 